\title{
El valor del idioma español en ciencia y tecnología
}

\author{
The Value of the Spanish Language \\ in Science and Technology
}

Luis M. Plaza, Begoña Granadino y

ESTHER GARCÍA-CARPINTERO

RECIBIDO: 2 DE AGOSTO DE 2017 ACEPTADO: 22 DE NOVIEMBRE DE 2017

Instituto de Filosofía (IF)

Consejo Superior de Investigaciones Científicas (CSIC)

C/ Albasanz 26-28. Madrid, 28037. España

luis.plaza@cchs.csic.es (Orcid ID: 0000-0002-1927-4920)

begona.granadino@cchs.csic.es (Orcid ID: 0000-0002-0144-8638)

esther.carpintero@cchs.csic.es (Orcid ID: 0000-0002-7804-1449)

\author{
Mario Albornoz, Rodolfo Barrere y LaUtaro Matas \\ Red Iberoamericana de Indicadores de Ciencia y Tecnología (RICYT) \\ Av. Pueyrredón 538, 2cpo, 2. ${ }^{\circ}$ piso, C. Buenos Aires, 1032. Argentina \\ albornoz@ricyt.org (Orcid ID: 0000-0002-6678-8684) \\ rbarrere@ricyt.org (Orcid ID: 0000-0002-4299-5021) \\ Imatas@ricyt.org (Orcid ID: 0000-0003-0272-1592)
}

Resumen: Se analiza el peso específico del español como idioma principal en las revistas científicas editadas en España y en los países latinoamericanos, así como a través de la producción científica mundial recogida en las principales bases de datos bibliográficas de cobertura internacional. El estudio pone de manifiesto la importancia del español como lengua de comunicación científica en determinados ámbitos disciplinares ligados por una parte a intereses socioeconómicos y territoriales de los países de habla española, como es el caso de la medicina clínica, la geología y medio ambiente y, por otra, a determina- dos ámbitos científicos propios de las ciencias sociales y las humanidades, como es el caso de la economía regional, la literatura, la historia de los países iberoamericanos, etc. Este estudio pretende contribuir al mejor conocimiento y comprensión del valor real y potencial del español en el mundo científico, de cara a la adecuada utilización de este recurso para el desarrollo de nuestras sociedades, y su proyección cultural y económica a escala internacional.

Palabras clave: Idioma español. Investigación científica. Tecnologías. Revistas científicas. 
Abstract: We analyse the specific weight of the Spanish as a primary language in scientific journals published in Spain and Latin American countries, as well as through the global scientific output covered by the main bibliographic databases of international coverage. The study highlights the importance of the Spanish as a language of scientific communication in certain disciplinary areas linked on the one hand to socio-economic regional interests of Spanish-speaking countries, as it is the case of Clinical medicine, Geology and Environment, and, on the other, to certain scientific fields of the Social sciences and Humanities, as it is the case of the Regional economy, the Literature, the History of Ibero-American countries, etc. This study aims to contribute to the better knowledge and understanding of the real and potential value of the Spanish in the scientific world, with regard to the appropriate use of this resource for the development of our societies, and its cultural and economic projection at the international level.

Keywords: Spanish Language. Scientific Research. Technologies. Scientific Journals.

\section{INTRODUCCIÓN Y OBJETIVOS}

a determinación del interés económico de la lengua española y su puesta en valor conlleva necesariamente el análisis del papel desempeñado por esta en todos aquellos sectores clave en el desarrollo socioeconómico de los países de habla española (Plaza; Plaza/Bordons). Los ámbitos de la ciencia y la tecnología, además de estar íntimamente interrelacionados, son en sí mismos un cruce de caminos donde los avances de la investigación científica y el desarrollo de las tecnologías constituyen el eje vertebral de la sociedad basada en el conocimiento, con las implicaciones que son evidentes en sectores estratégicos, como el industrial, sanitario, medioambiental y educativo, entre otros (BOE).

Este estudio tiene por objetivo fundamental determinar el peso específico del español en la comunicación de los resultados de la investigación científica realizada en lengua española, a través de su presencia en el contexto científico internacional.

Se pretende que los resultados de este estudio contribuyan al análisis en profundidad del papel que desempeña la lengua española en distintos ámbitos disciplinares de la ciencia y la tecnología y que los resultados obtenidos permitan conocer las características cuantitativas y cualitativas más relevantes que definen el papel del español como lengua de comunicación científica y tecnológica (FECYT).

\section{Metodología}

Este estudio conlleva, por una parte, un análisis de la producción de revistas científicas editadas en España y en países latinoamericanos (Alonso Gamboa/Russell), y por otra un análisis de la producción científica, en español y en 
otras lenguas, recogida tanto en revistas editadas en países de habla española como en revistas extranjeras de amplia proyección internacional. La expresión "producción científica" hace alusión al conjunto de artículos científicos y otras contribuciones publicadas en revistas científicas.

Fuentes de información. Para el análisis de revistas científicas editadas en España y en los países de América Latina, la obtención de datos se ha realizado mediante consulta al Directorio Latindex, que es fruto de la cooperación de una red de instituciones científicas y académicas que de manera coordinada reúnen y diseminan información bibliográfica sobre las publicaciones científicas seriadas producidas en América Latina, Caribe, España y Portugal. El análisis de la producción científica en lengua española se ha llevado a cabo utilizando las bases de datos del CSIC y una serie de bases de datos bibliográficas de cobertura internacional.

La producción científica en Ciencias Experimentales se analiza, por una parte, a través de la base de datos ICYT, que cubre revistas sobre temáticas de interés territorial o regional español. Por otra parte, la producción científica de amplia proyección internacional se analiza a través de las bases de datos Web of Science, Scopus e Inspec.

El análisis de la producción científica en Ciencias de la Salud se lleva a cabo a partir de bases de datos WoS-Science Citation Index Expanded, producida por Clarivate Analytics (en sus apartados de Medicine, Pharmacology, Nursery), Scopus, producida por Elsevier y PubMed (que incluye Medline), producida por el US National Institute of Health, las cuales permiten obtener una visión muy representativa del español en disciplinas médicas y sanitarias de proyección internacional, mientras que los datos obtenidos a través de la base de datos IME (Índice Médico Español), producida conjuntamente por el CSIC y la Facultad de Medicina de la Universidad de Valencia, permiten obtener una visión complementaria y focalizada en la investigación de carácter clínico publicada en revistas españolas de alcance nacional.

El análisis de la producción científica en Disciplinas Tecnológicas se realiza a partir de las bases de datos del CSIC (base de datos ICYT: Índice Español de Ciencia y Tecnología) y de la base de datos Inspec, de cobertura internacional, producida por The Institution of Engineering and Technology del Reino Unido.

Para Ciencias Sociales se han utilizado el WoS-Social Sciences Citation Index, Scopus, y la base de datos ISOC (Índice Español de Ciencias Sociales y Humanidades), producida por el CSIC (Abejón Peña y otros). 
Para el caso de las Humanidades se han utilizado las bases de datos $\mathrm{WoS}_{0}$ Arts \& Humanities Citation Index, Scopus y la mencionada base de datos ISOC.

La metodología seguida conlleva la obtención y análisis de indicadores de producción científica correspondientes a artículos publicados en español y su comparación frente a la producción científica publicada en inglés y en otras lenguas.

El análisis de producción científica comprende el periodo 2005-2010, por lo que se consideran los artículos que han sido publicados en dicho intervalo de tiempo. Por otra arte, la información obtenida mediante consulta a $\mathrm{La}$ tindex corresponde a enero de 2014.

\section{RESULTADOS}

La producción de revistas científicas editadas en España y en países latinoamericanos

$\mathrm{Al}$ margen de las revistas de corriente principal (indexadas en SCI y SCOPUS), existe una importante producción científica en Iberoamérica que se comunica mediante otros canales y donde el idioma español tiene una gran importancia. Latindex es una fuente de información clave para dar cuenta de este fenómeno. Este directorio es fruto de un esfuerzo regional que recopila datos sobre revistas científicas en todas las áreas del conocimiento. Las revistas cubiertas por Latindex son evaluadas según una serie de criterios de calidad (33 para revistas impresas, 36 para revistas electrónicas). Según el número de criterios

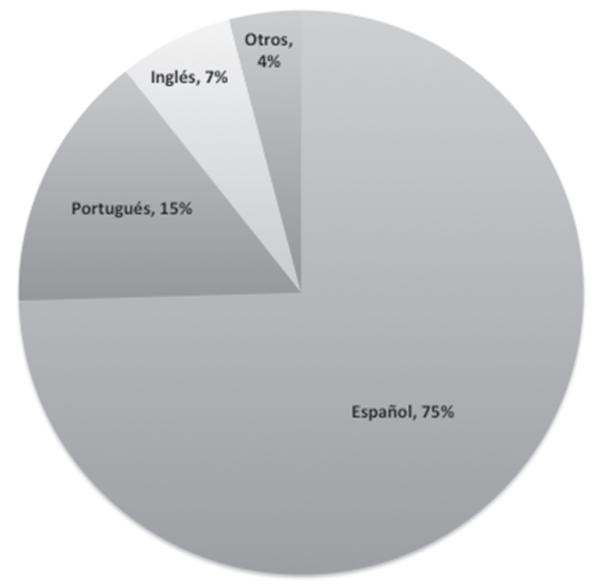

Figura 1. Distribución porcentual de las revistas en el Catálogo de Latindex según idioma de publicación 
que las publicaciones cumplen, se constituyen dos grupos de revistas. El Directorio, con 22406 publicaciones, y el Catálogo, con 7377. De estas últimas, que cuentan con un control de calidad más riguroso, 6979 están actualmente vigentes. Sobre ese grupo, con datos obtenidos en enero de 2014, se ha realizado esta parte del estudio. Las revistas vigentes del Directorio cumplen, en promedio, con el $91 \%$ de los criterios de calidad Latindex.

Un $75 \%$ de las revistas cubiertas por Latindex se editan en español (5203 títulos) y un $15 \%$ en portugués (1031 títulos). Un número significativo de revistas (459) se publica en inglés, lo que supone un $7 \%$ del total (fig. 1).

Considerando el país de edición de las revistas, la mayor parte son brasileñas (1943); las españolas forman el segundo grupo (1878). A continuación aparecen las revistas editadas en México (727) y Argentina (524). Esta distribución resulta consistente con el tamaño relativo de sus sistemas científicos, medidos a través de otros indicadores. Estos cuatro países reúnen el 73 \% de las revistas registradas (fig. 2).

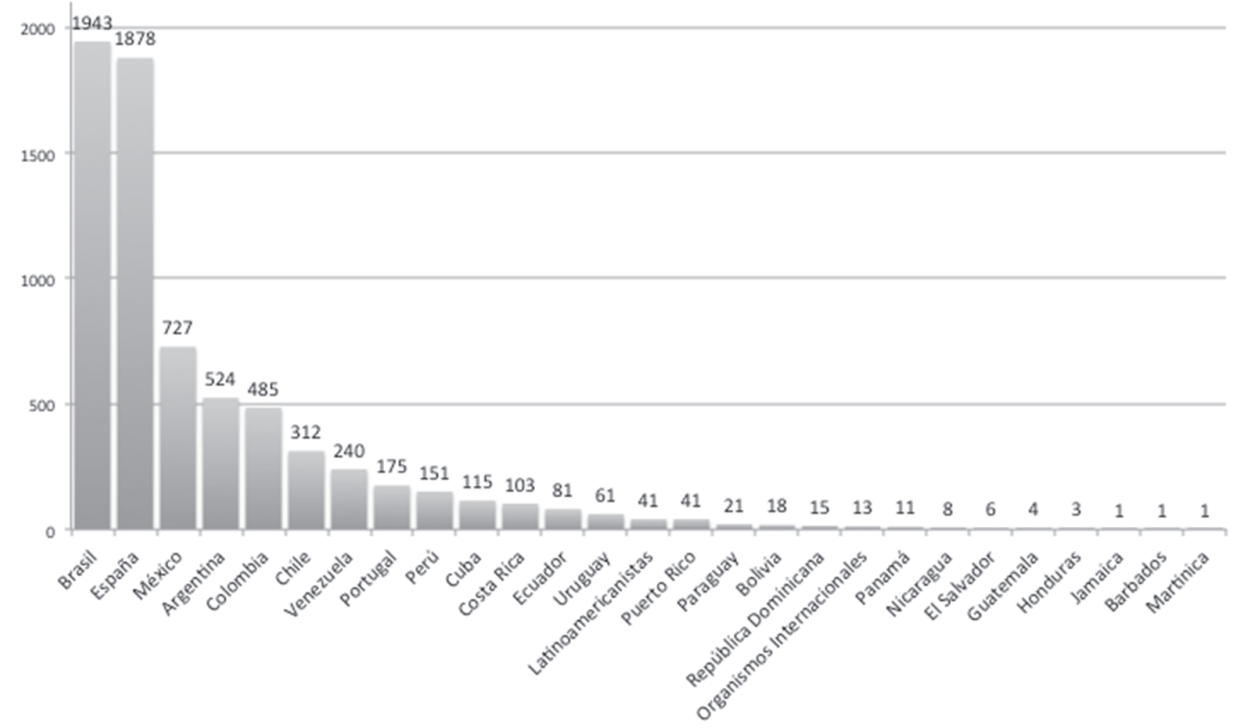

Figura 2. Distribución de las revistas del Catálogo de Latindex por país de edición

Dentro del Catálogo de Latindex existe una fuerte presencia de revistas de Ciencias Sociales (36 \%) y de Humanidades (24 \%). Luego aparecen las Ciencias Médicas (15\%). Las Ciencias Exactas y Naturales, mejor representadas 
en las bases de corriente principal, agrupan aquí solo el $11 \%$ de las publicaciones. Las Ciencias Agrícolas un $4 \%$ y las Ingenierías y Tecnologías el $3 \%$ (fig. 3).

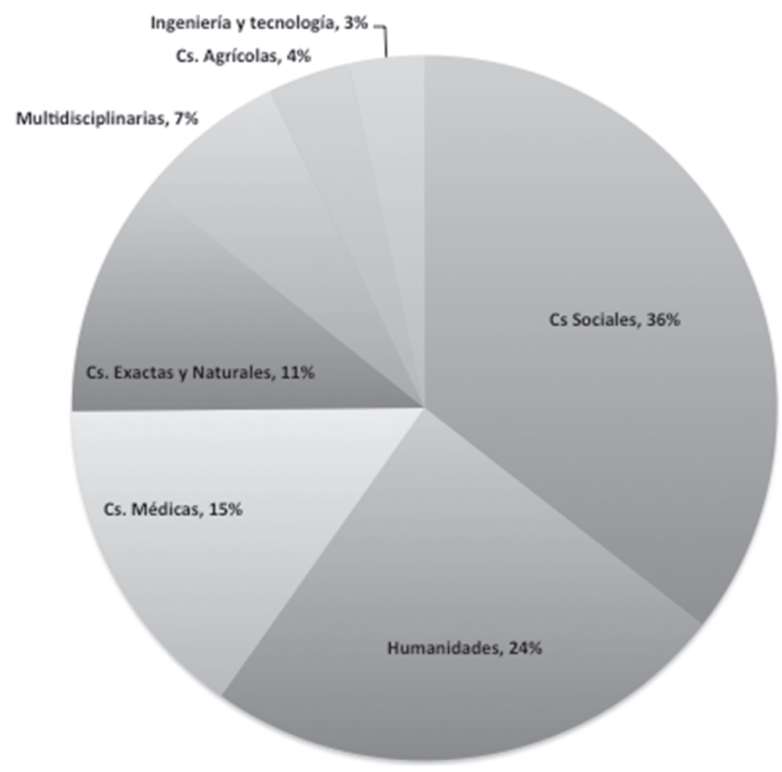

Figura 3. Distribución de las revistas del Catálogo de Latindex por disciplina científica

La presencia del idioma español distribuido por disciplinas científicas es homogénea, situándose ligeramente por encima del $75 \%$ del total de las revistas de cada área temática. La excepción son las revistas multidisciplinares, donde solo el $67 \%$ de las publicaciones se editan en español. En las Ciencias Sociales y Humanidades la publicación en inglés solo alcanza el $3 \%$. Esto pone en evidencia que estas disciplinas tienen canales diferentes de comunicación, en los que otros idiomas, como el portugués y el español, tienen un papel importante como transmisores de conocimiento científico en los distintos ámbitos regionales. Sí existe un patrón diferenciado en las revistas editadas en inglés, que son significativamente más frecuentes en las Ciencias Experimentales. En las Ciencias de la Salud, las Ciencias Exactas y las Ciencias Naturales, las disciplinas Agrícolas y las Ingenierías y Tecnologías, la publicación en inglés abarca -en promedio- el $13 \%$ de las revistas. 
Es interesante observar cómo el incremento de la presencia del inglés en las Ciencias Experimentales va en detrimento de la publicación en portugués. El caso paradigmático es el de las Ciencias Exactas y Naturales, donde la presencia del portugués es de tan solo un $8 \%$ cuando en el total de las revistas del Catálogo de Latindex es del $15 \%$. Sin embargo, la presencia del idioma español no se ve afectada por este leve incremento del uso del inglés como idioma de comunicación en estas revistas (fig. 4).

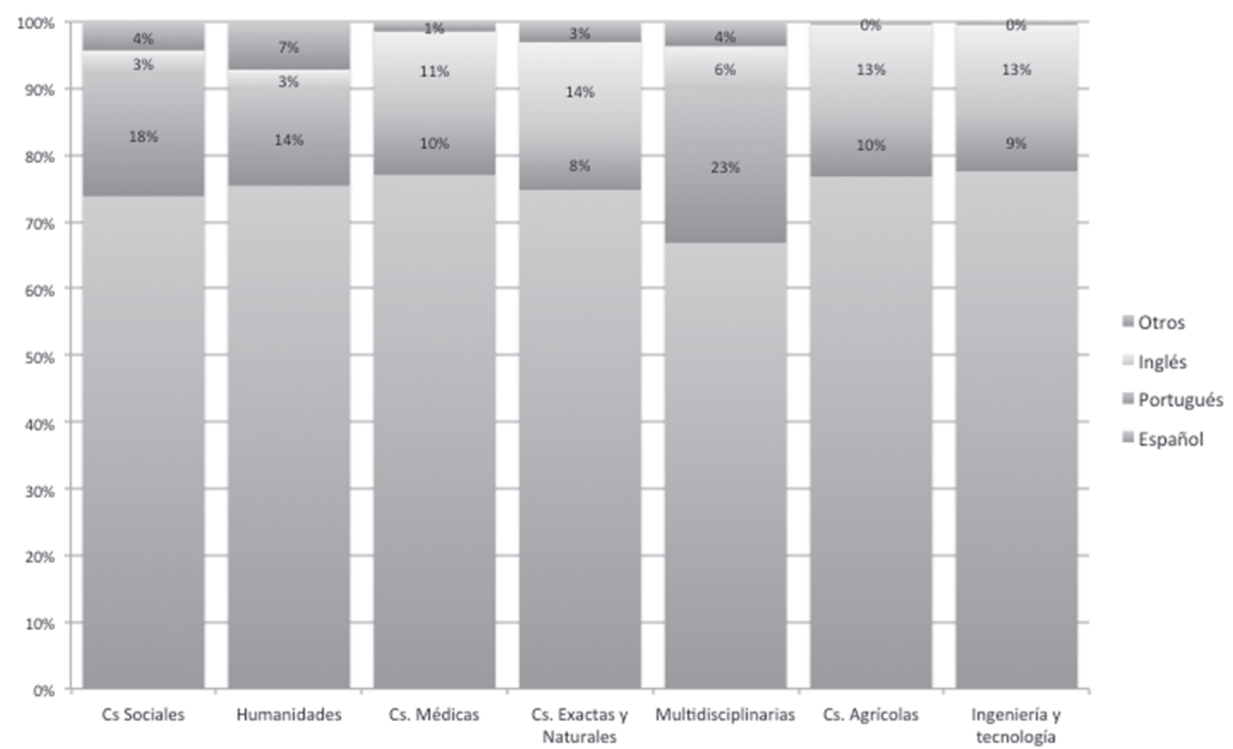

Figura 4. Distribución de las revistas del Catálogo de Latindex por idioma de publicación y disciplina

La distribución por países de las revistas editadas en inglés confirma este dato (fig. 5). Destaca el caso de Portugal, donde el $29 \%$ de las revistas registradas en el Catálogo de Latindex editadas en ese país se publica en inglés. En Brasil el $16 \%$ de las revistas que se editan (registradas en el Catálogo de Latindex) se publica en inglés.

En los países hispanoparlantes, en cambio, el porcentaje de revistas editadas en inglés es mucho menor. En España alcanza el 3,6 \%, seguido de Chile con el 2,9\%. En el resto de los países, la proporción está en todos los casos por debajo del $2 \%$. 


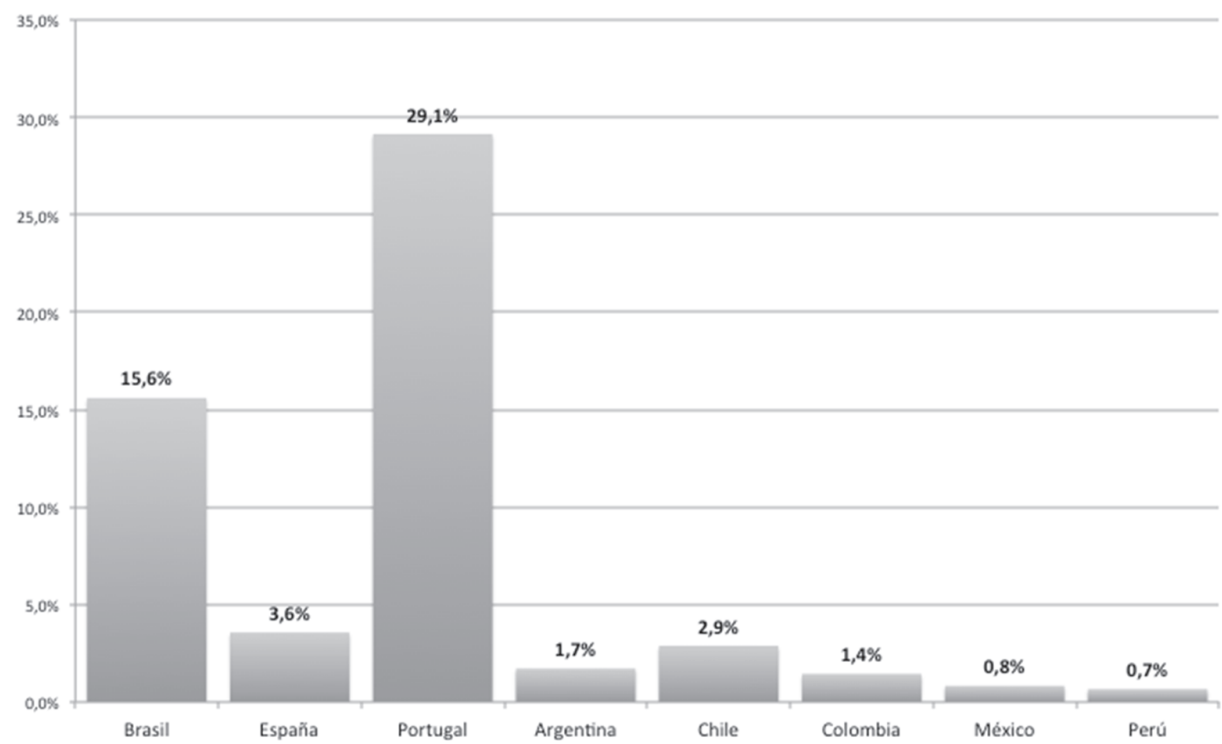

Figura 5. Porcentaje de las revistas del Catálogo de Latindex editadas en inglés en relación con el total de revistas de cada país

La producción científica en español: principales indicadores a escala nacional

Este apartado recoge los indicadores de producción científica recopilados en revistas españolas durante el periodo 2005 a 2010.

La producción científica en Ciencias Experimentales y Tecnologías durante el periodo analizado es de 31714 documentos (fundamentalmente artículos), de los cuales 23112 (72,8\%) han sido publicados en español, frente a 7620 (24 \%) en inglés. En Ciencias Sociales y Humanidades, la producción científica en este mismo periodo es de 121434 documentos, de los que 106295 (87,5\%) han sido publicados en español, frente a 5896 (4,8 \%) en inglés. En Medicina Clínica y otras disciplinas de interés sanitario, cubiertas por la base de datos IME, la producción científica en el periodo objeto de estudio es de 35484 documentos, de los que 33804 (95,2\%) han sido publicados en español, frente a tan solo 1609 (4,5\%) publicados en inglés (fig. 6).

Un análisis del balance entre los documentos publicados en español e inglés a lo largo de estos años permite afirmar que, en Ciencias Experimentales, se ha ido incrementando de forma gradual el porcentaje de artículos en inglés, 


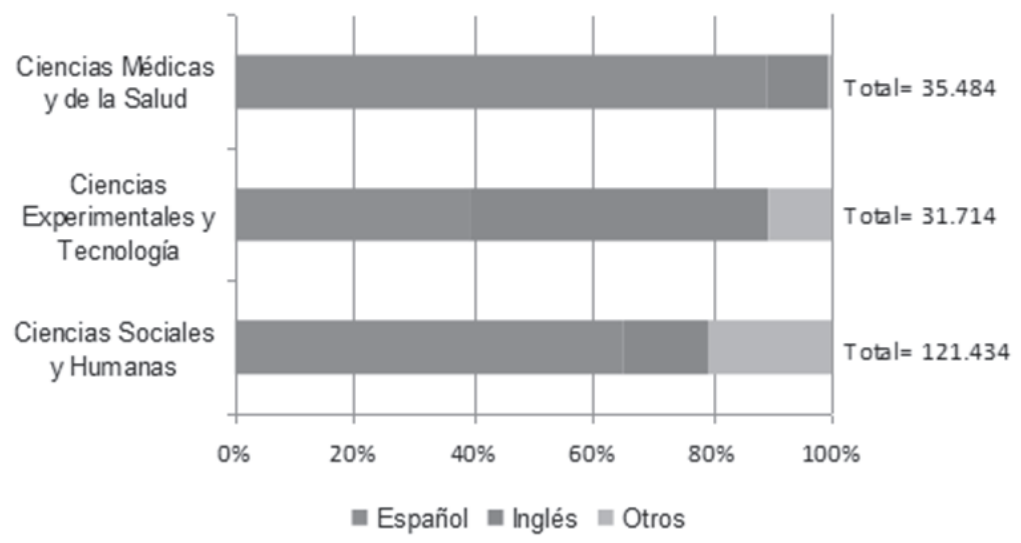

Figura 6. Producción científica en revistas españolas

mientras que el español ha ido disminuyendo proporcionalmente, aunque en la actualidad siga siendo la lengua más utilizada por los autores que publican en las revistas españolas. Este mismo hecho se está produciendo en el ámbito de las Ciencias Sociales y las Humanidades, aunque de forma mucho menos acusada (fig. 7).

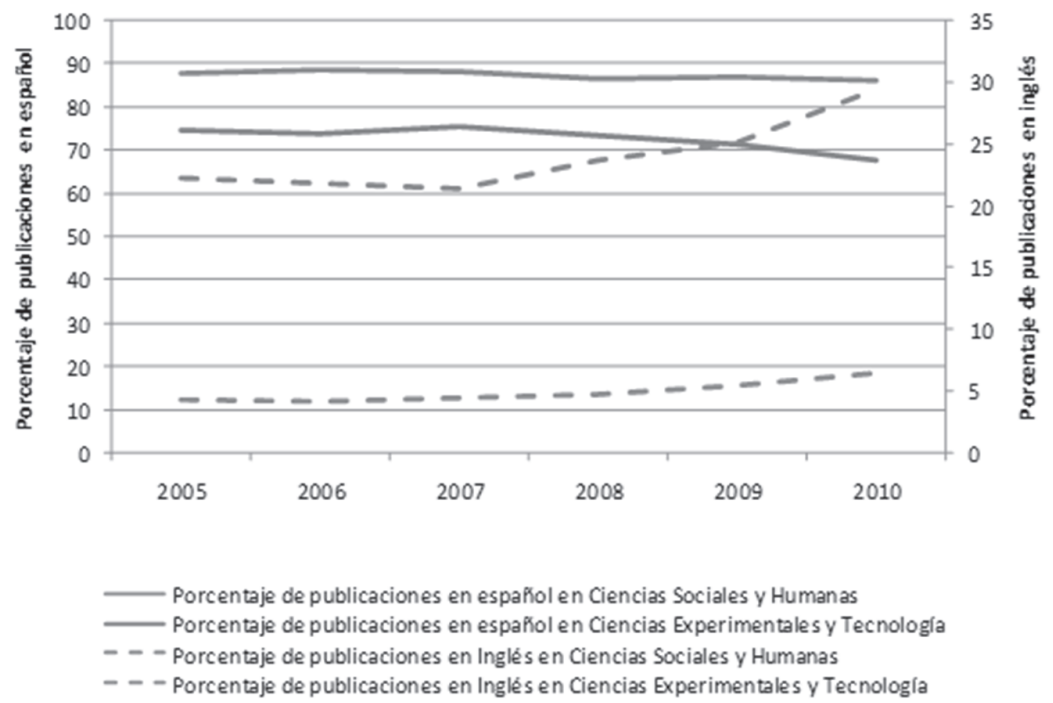

Figura 7. Evolución de la producción científica en Ciencias experimentales y Ciencias Sociales en español e inglés 
El análisis de la información representada en estas figuras pone de relieve varios hechos. En primer lugar, la acusada sobre-representación del español en el ámbito de la Medicina Clínica y Ciencias de la Salud. Los valores representados en la figura vienen a confirmar que este tipo de publicaciones están mayoritariamente dirigidas a médicos y personal sanitario ligado a la investigación y a la práctica clínica, entornos en los que el uso del inglés no está particularmente extendido.

En segundo lugar, la también esperada sobre-representación del español en las publicaciones correspondientes al ámbito de las Humanidades y las Ciencias Sociales. En casi todos estos ámbitos disciplinares, el uso del español como lengua de publicación ha venido siendo, y es aún hoy en día, la pauta general entre los investigadores españoles. Solo muy recientemente, influenciados por una creciente internacionalización de la investigación en estas disciplinas y por la creciente presión que ejercen los mecanismos de evaluación de la actividad investigadora, se empieza a hacer visible un cambio de tendencia hacia la publicación en inglés. El balance entre español e inglés en algunas disciplinas de las Ciencias Sociales es mucho más equilibrado, e incluso decididamente orientado al uso del inglés, como en los casos de la Economía, la Sociología y los Estudios cienciométricos y bibliométricos.

La producción científica en español: principales indicadores a escala internacional: análisis a través de la Web of Science

A continuación, se muestran los resultados del análisis de presencia del español en la producción científica mundial recogidos en las principales bases de datos bibliográficas internacionales accesibles a través de la Web of Science (WoS): Sience Citation Index Expanded (SCI-Expanded), Social Sciences Citation Index (SSCI) y Arts and Humanities Citation Index (AHCI). Como se resume en la tabla, el español es una lengua de muy limitada presencia en estas bases de datos, con valores que, en términos porcentuales, van desde el 0,24 \% de la producción científica en disciplinas experimentales, al 2,4 \% de la producción científica en Artes y Humanidades. Considerando que estos valores se han obtenido a partir de una colección compuesta por cerca de 9 millones de registros bibliográficos, cabe concluir que el español es una lengua de muy limitada proyección entre las denominadas "revistas de corriente principal", que son las que componen los fondos documentales de estas bases de datos. 


\begin{tabular}{|c|c|c|c|c|c|}
\hline BASE DE DATOS & \multicolumn{2}{|c|}{ DOCUMENTOS EN INGLÉS } & \multicolumn{2}{|c|}{ DOCUMENTOS EN ESPAÑOL } & DOCUMENTOS TOTALES \\
\hline sCl-Expanded & 7035877 & $(96,44 \%)$ & 17727 & $(0,24 \%)$ & 7296077 \\
\hline$S S C l$ & 1098872 & $(94,00 \%)$ & 11345 & $(1,00 \%)$ & 1168420 \\
\hline $\mathrm{AHCl}$ & 48992 & $(72,20 \%)$ & 16424 & $(2,40 \%)$ & 678704 \\
\hline Total wos & 10289863 & & 47367 & & 10838268 \\
\hline
\end{tabular}

Tabla 1. Número de documentos por base de datos de la Web of Science

\section{Science Citation Index Expanded (SCI)}

La base de datos Science Citation Index Expanded indiza 8300 revistas de 150 disciplinas científicas. Se ha analizado el idioma de los 7296077 documentos recogidos durante el periodo 2005-2010. El 97 \% de estos documentos están publicados en inglés (fig. 8). El resto de los idiomas identificados representa un 3,6 \% de los documentos, siendo el alemán el idioma más utilizado después del inglés con un porcentaje inferior al $1 \%$. El español es el quinto idioma más utilizado lo que supone un $0,24 \%$ del total de las publicaciones recogidas en el periodo analizado.

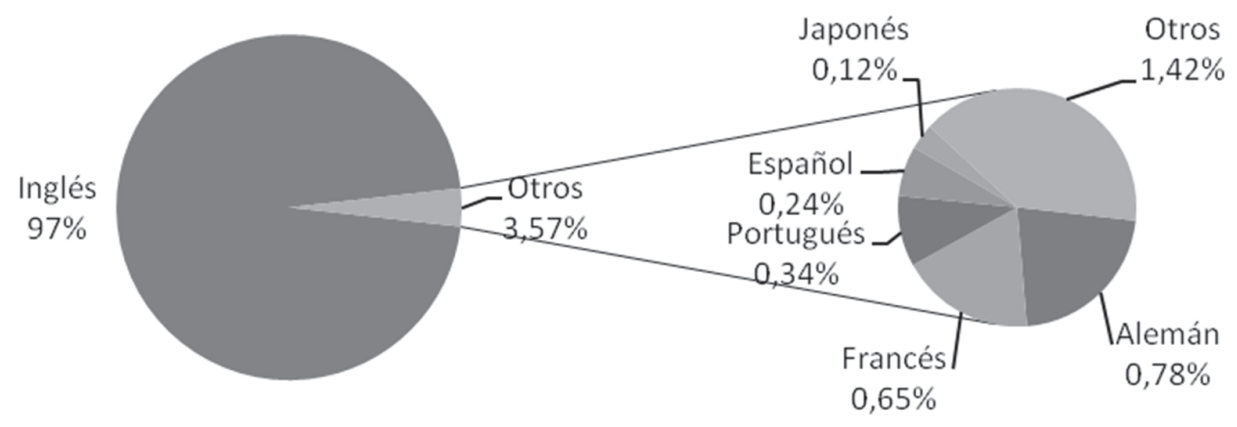

Figura 8. Idiomas de publicación en Science Citation Index Expanded. Periodo 2005-2010

El porcentaje de publicaciones en español en la base de datos SCI muestra un comportamiento irregular, con una primera etapa de crecimiento entre 2006 y el 2008, seguida por otra etapa de decrecimiento durante el periodo 2008-2010 (fig. 9). Sin embargo, la evolución del porcentaje de publicaciones en inglés muestra una clara tendencia negativa a lo largo de todo el periodo (fig. 10). 


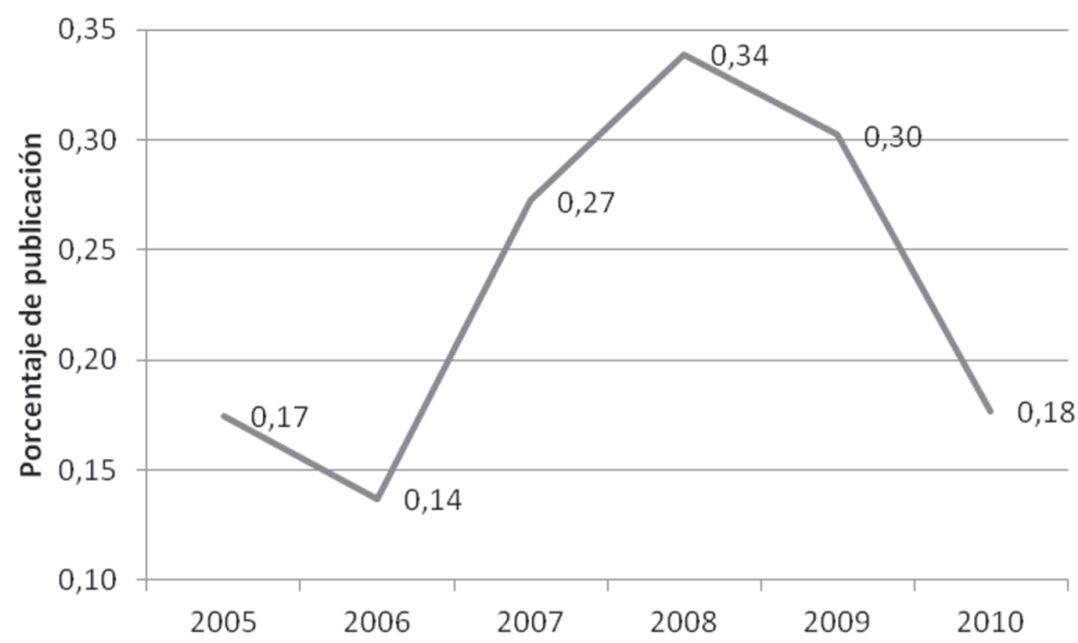

Figura 9. Evolución del porcentaje de publicaciones en español recogidas en Science Citation Index Expanded. Periodo 2005-2010

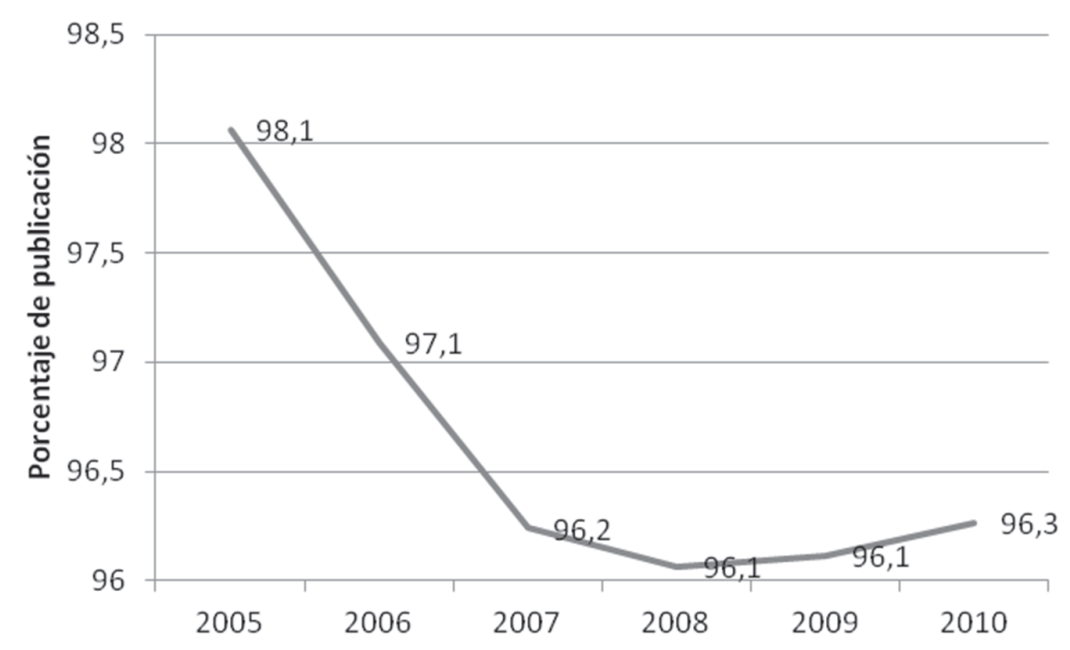

Figura 10. Evolución en el porcentaje de publicaciones en inglés recogidas en Science Citation Index Expanded. Periodo 2005-2010

El área temática con más publicaciones en español es la disciplina de Medicina Interna, que representa un $19 \%$ de todas las publicaciones en español recogidas en el SCI en este periodo; representa un 1,7\% del total de las publicaciones en esta disciplina. La categoría donde el español alcanza un mayor por- 
centaje de representación es Música, con un 2,87 \% del total de documentos recogidos en SCI. Sin embargo, el área de Música solo representa un 0,078 \% de los documentos en español recogidos en SCI.

\section{Social Sciences Citation Index (SSCI)}

La base de datos Social Sciences Citation Index indiza 4500 revistas de 50 disciplinas de las Ciencias Sociales. Durante el periodo 2005-2010 se indizaron 1168420 documentos, siendo el inglés el idioma predominante, con un $94 \%$ de las publicaciones. El resto de idiomas representan un $6 \%$ de los documentos. El alemán es el segundo idioma más utilizado, con un $1,9 \%$ de los documentos. En esta categoría el español adquiere un mayor peso pasando a ser el tercer idioma, con un 1,0 \% de todos los documentos (fig. 11).

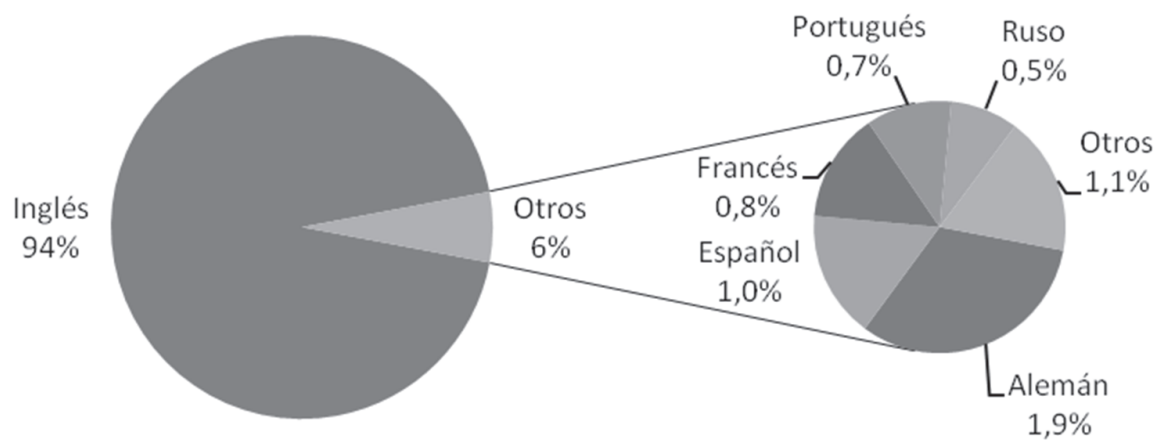

Figura 11. Idiomas de publicación en Social Sciences Citation Index durante el periodo 2005-2010

La evolución del español en los trabajos publicados en las revistas cubiertas por la base de datos SSCI es muy parecida a la observada en la base de datos SCI, con un periodo de crecimiento hasta el 2008. A partir de esa fecha, se observa un periodo de decrecimiento. En el caso del idioma inglés se observa una tendencia de decrecimiento en el número de publicaciones hasta 2010, cuando se observa un aumento en el porcentaje de publicaciones en inglés (figs. 12 y 13). 
PLAZA. EL VALOR DEL IDIOMA ESPAÑOL EN CIENCIA Y TECNOLOGÍA

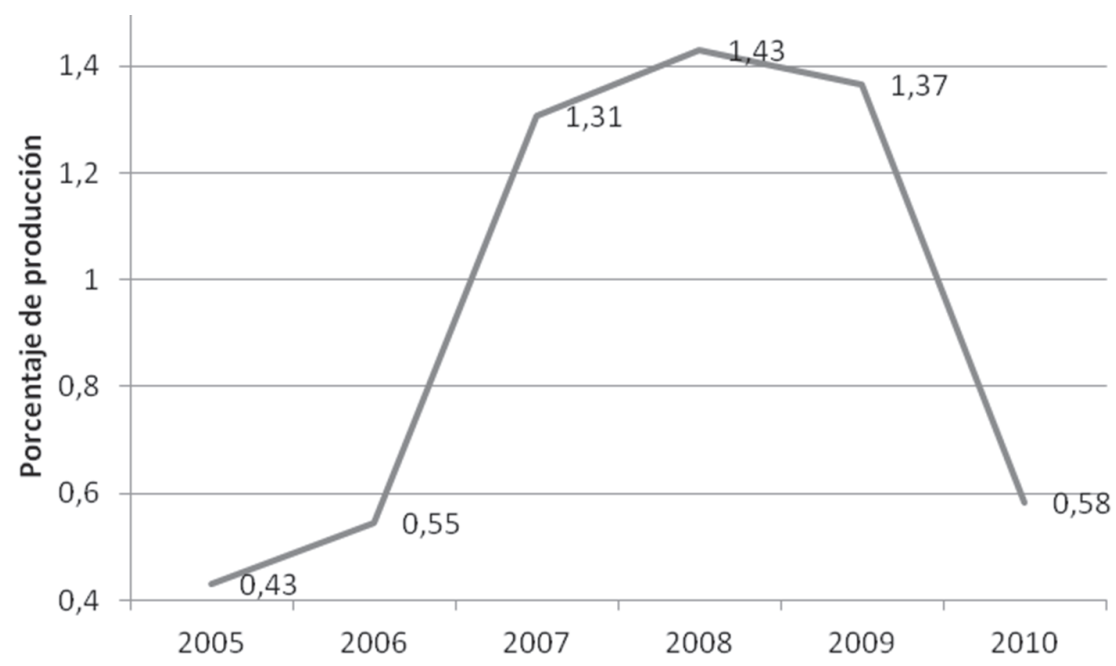

Figura 12. Evolución del porcentaje de publicaciones en español recogidas en Social Science Citation Index. Periodo 2005-2010

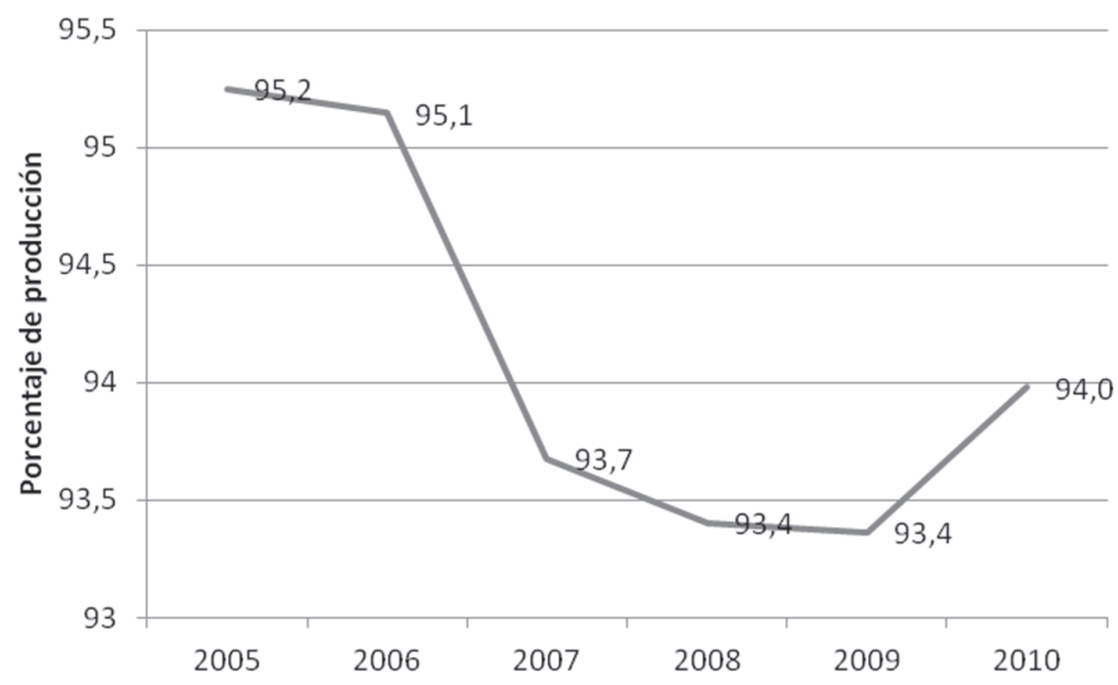

Figura 13. Evolución del porcentaje de publicaciones en inglés recogidas en Social Science Citation Index. Periodo 2005-2010

La disciplina con más publicaciones en español es la Psicología y representa un $16 \%$ de todas las publicaciones en español recogidas en esta base de datos durante el periodo analizado. 


\section{Arts and Humanities Citation Index}

En la base de datos Arts \& Humanities Citation Index se indizan 2300 revistas del área del Arte y las Humanidades. El total de documentos recogidos en el periodo 2005-2010 es de 678 704. En esta base de datos el inglés pierde representación comparado con los datos del SCI y SSCI a favor del francés, que pasa a ser el segundo idioma con mayor representación con un $11 \%$ de los documentos. El español ocupa la quinta posición con un $2 \%$ de los documentos (fig. 14).

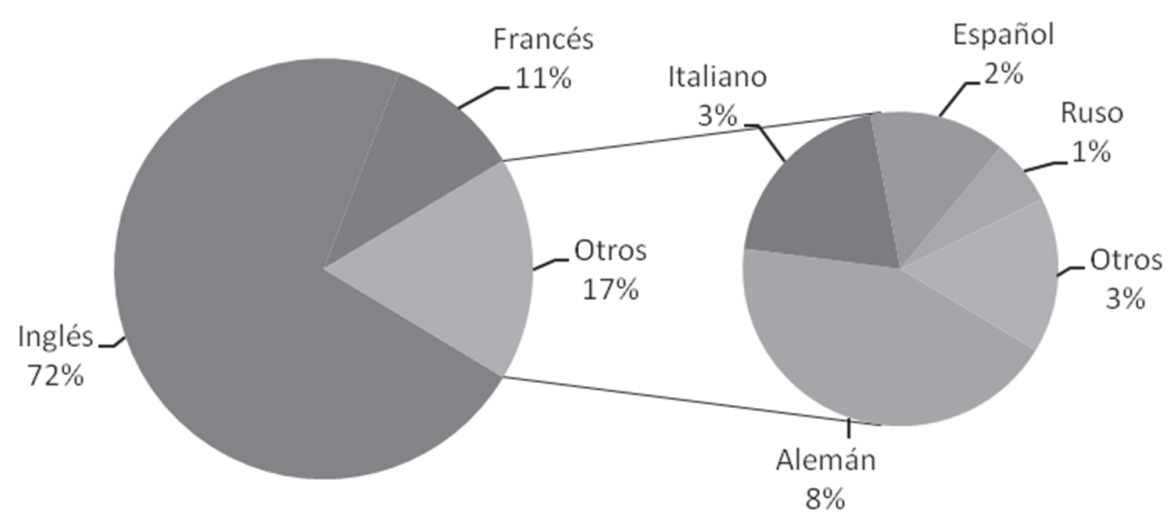

Figura 14. Idiomas de publicación en Arts and Humanities Citation Index. Periodo 2005-2010

La evolución del español durante el periodo estudiado muestra un aumento en el número de publicaciones hasta 2010, cuando decrece drásticamente. El inglés muestra dos etapas diferenciadas, una de decrecimiento durante el periodo 2005-2007 y otra de crecimiento desde el 2008 hasta el final del periodo (figs. 15 y 16). La disciplina con más publicaciones en español es la Literatura, que representa un $36,2 \%$ de todas las publicaciones en español recogidas en esta base de datos en el periodo analizado. 
PLAZA. EL VALOR DEL IDIOMA ESPAÑOL EN CIENCIA Y TECNOLOGÍA

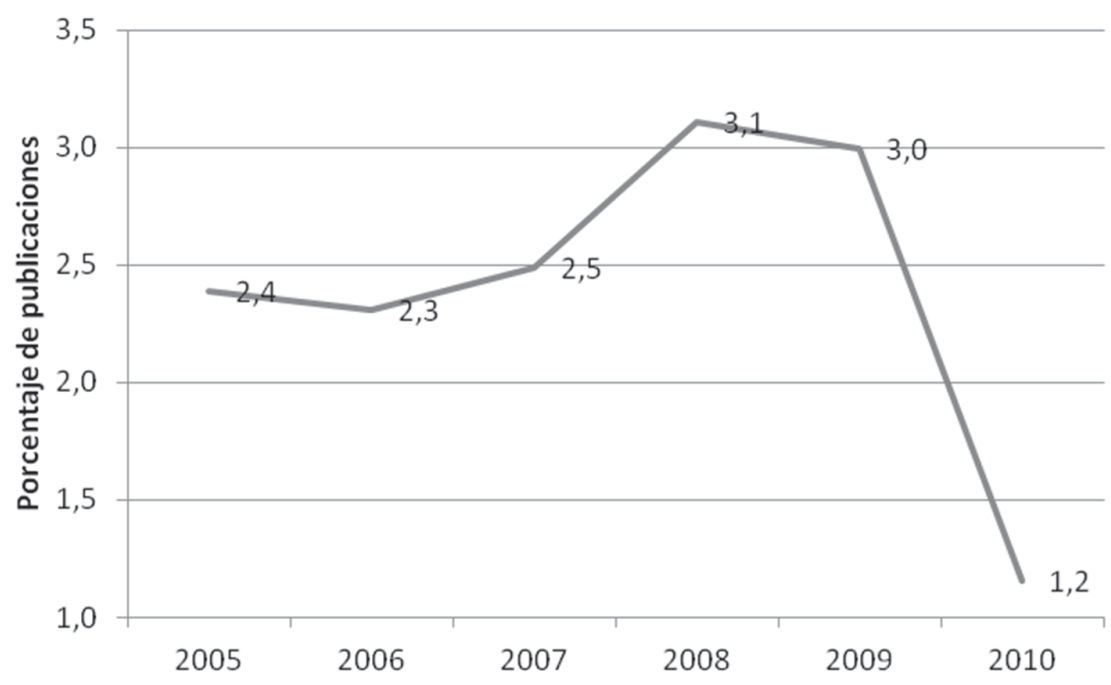

Figura 15. Evolución del porcentaje de publicaciones en español recogidas en Arts and Humanities Citation Index. Periodo 2005-2010

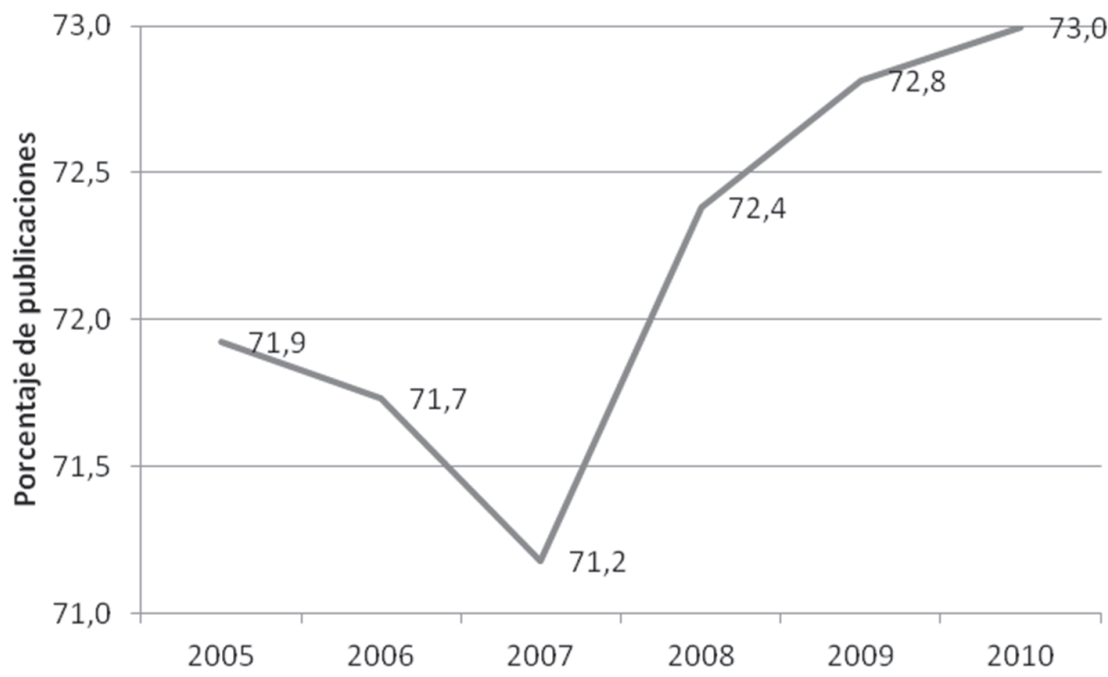

Figura 16. Evolución del porcentaje de publicaciones en inglés recogidas en Arts and Humanities Citation Index. Periodo 2005-2010 
La producción cientifica en español: principales indicadores a escala internacional: análisis a través de Scopus

Scopus es una base de datos internacional que recoge 18500 revistas. Está dividida en cuatro grandes áreas: Ciencias de la Vida, Ciencias de la Salud, Ciencias Físicas y Ciencias Sociales. A su vez, estas áreas se dividen en distintas disciplinas. Esta base de datos está más centrada en publicaciones de origen europeo frente a Web of Science más orientada hacia publicaciones de EE.UU. y Reino Unido. Esto hace que el inglés, aunque sigue siendo el idioma más utilizado en las publicaciones recogidas en Scopus durante el periodo 2005-2010, tenga un menor peso a favor de otros idiomas como el alemán, el francés e incluso el español, que llega a superar hasta en más de un $400 \%$ las publicaciones en español recogidas en WoS. Sin embargo, en el área de Ciencias Físicas sigue siendo prácticamente el único idioma de publicación, con más de un $95 \%$ de las publicaciones. El mayor número de publicaciones en español recogidas en Scopus corresponde al área de Ciencias de la Salud (tabla 2), que supone un $62 \%$ de las publicaciones en español.

\begin{tabular}{|c|c|c|c|c|c|}
\hline ÁREA & \multicolumn{2}{|c|}{ PUBLICACIONES EN INGLÉS } & \multicolumn{2}{|c|}{ PUBLICACIONES EN ESPAÑOL } & $\begin{array}{l}\text { NÚMERO TOTAL } \\
\text { DE PUBLICACIONES }\end{array}$ \\
\hline Ciencias de la Salud & 1839808 & $(79,0 \%)$ & 302216 & $(1,3 \%)$ & 23641055 \\
\hline Ciencias de la Vida & 22107762 & $(80,0 \%)$ & 314213 & $(1,1 \%)$ & 27622263 \\
\hline Ciencias Físicas & 5566707 & $(92,5 \%)$ & 14875 & $(0,3 \%)$ & 6016765 \\
\hline Ciencias Sociales & 1350808 & $(87,2 \%)$ & 25032 & $(1,6 \%)$ & 1539024 \\
\hline Total & 12363181 & $(88,6 \%)$ & 216106 & $(1,5 \%)$ & 13948907 \\
\hline
\end{tabular}

Tabla 2. Número de documentos por áreas de la base de datos Scopus

El segundo idioma más utilizado, después del inglés, es el alemán, que representa más del $5 \%$ de las publicaciones en la categoría de Ciencias de la Salud y de la Vida y un $1 \%$ en Ciencias Físicas (figs. 17, 18 y 19). El francés es el segundo idioma más utilizado en Ciencias Sociales, donde representa un $3,8 \%$ de las publicaciones recogidas en esta área, y es el tercero más utilizado en el resto de las tres áreas (fig. 20).

El peso específico del español, con respecto al inglés, en las publicaciones recogidas en Scopus durante el periodo 2005-2010 es muy pequeño. En ninguna de las cuatro áreas de esta base de datos llega a superar el 1,6 \% y no 
supera la quinta posición en Ciencias de la Salud, Ciencias de la Vida y Ciencias Físicas. En el caso de Ciencias Sociales, el español alcanza su mayor representación, con un 1,6\% de las publicaciones totales en esta área, y ocupa la cuarta posición por detrás del inglés, francés y alemán.

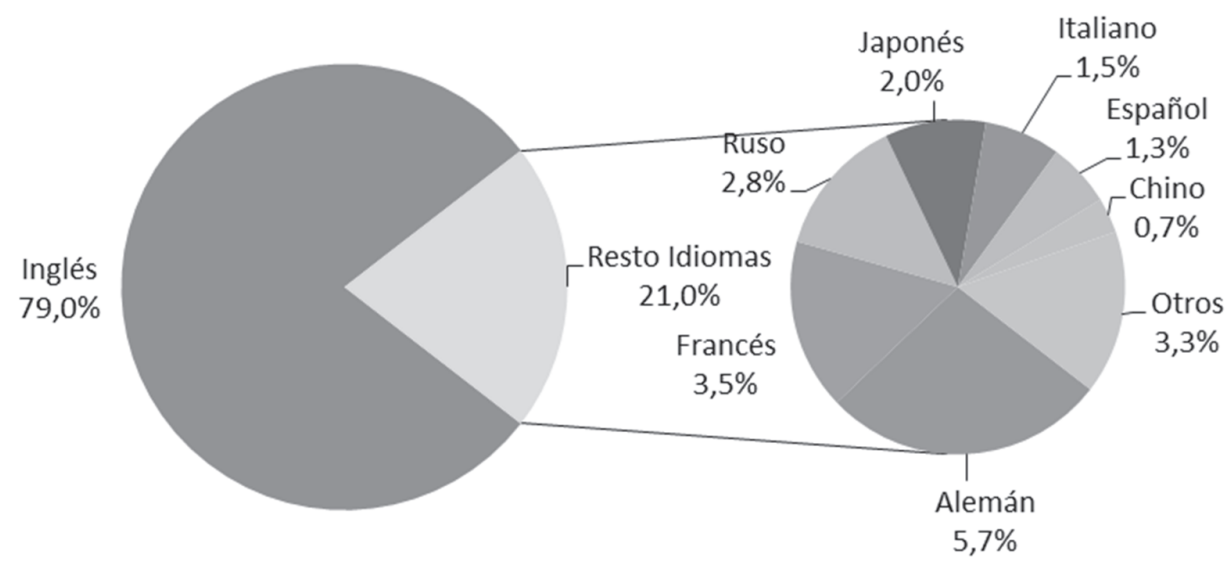

Figura 17. Idiomas representados en el área de Ciencias de la Salud de la base de datos Scopus. Periodo 2005-2010

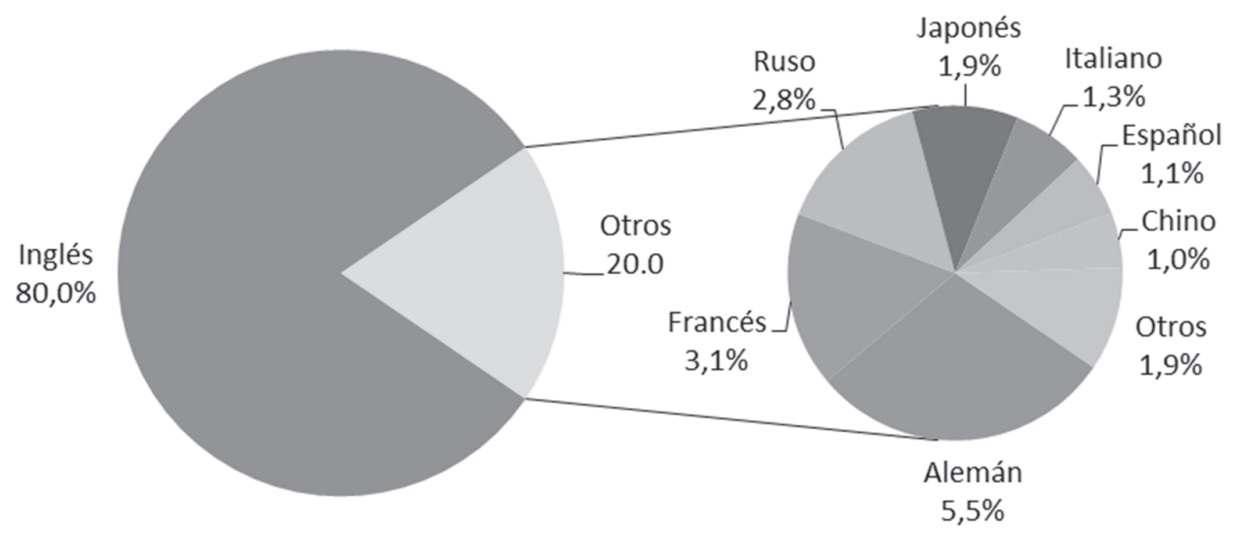

Figura 18. Idiomas representados en el área de Ciencias de la Vida de la base de datos Scopus. Periodo 2005-2010 


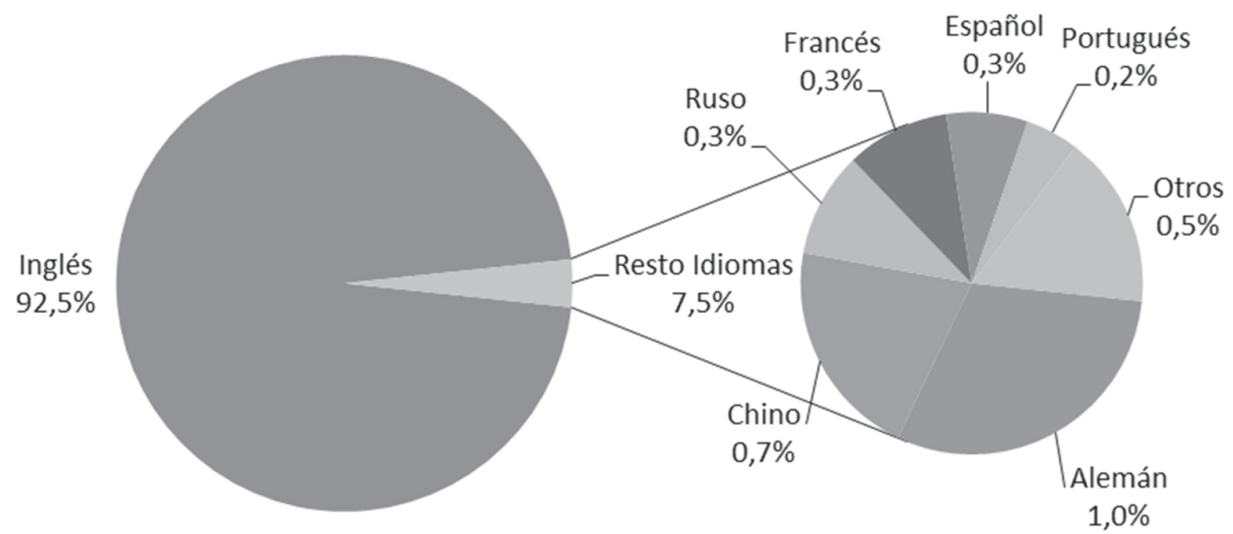

Figura 19. Idiomas representados en el área de Ciencias Físicas de la base de datos Scopus. Periodo 2005-2010

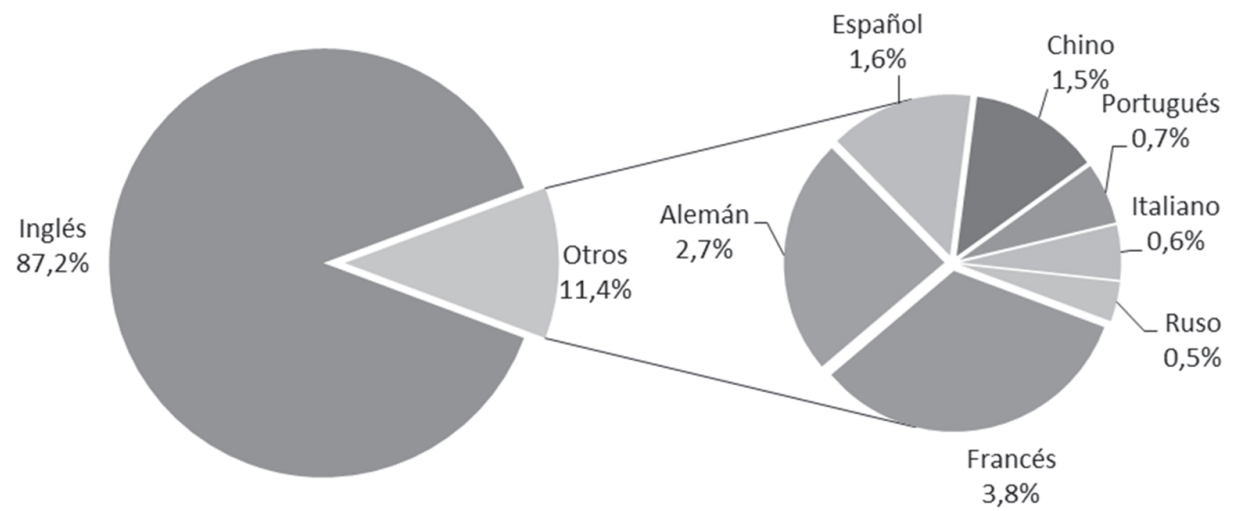

Figura 20. Idiomas representados en el área de Ciencias Sociales de la base de datos Scopus. Periodo 2005-2010

A lo largo del periodo estudiado, las publicaciones en español relacionadas con Ciencias Sociales experimentaron un aumento hasta 2009, pasando de representar un 1,3\% del total de publicaciones en esta área en 2005 hasta un $2 \%$ en 2009 (fig. 21). Las publicaciones en español en las áreas de Ciencias de la Salud y Ciencias de la Vida permanecen constantes a lo largo de todo el periodo estudiado. Cabe destacar la escasa representación del español en el área de Ciencias Físicas, donde no llega a superar el 0,3 \% de todas las publicaciones mundiales durante el periodo 2005-2010.

En el caso de la evolución de las publicaciones en inglés, se observa un ligero aumento en todas las áreas (fig. 22). El peso específico del inglés en el área de Ciencias de la Vida es muy alto, con una media anual del 92,5\%. 


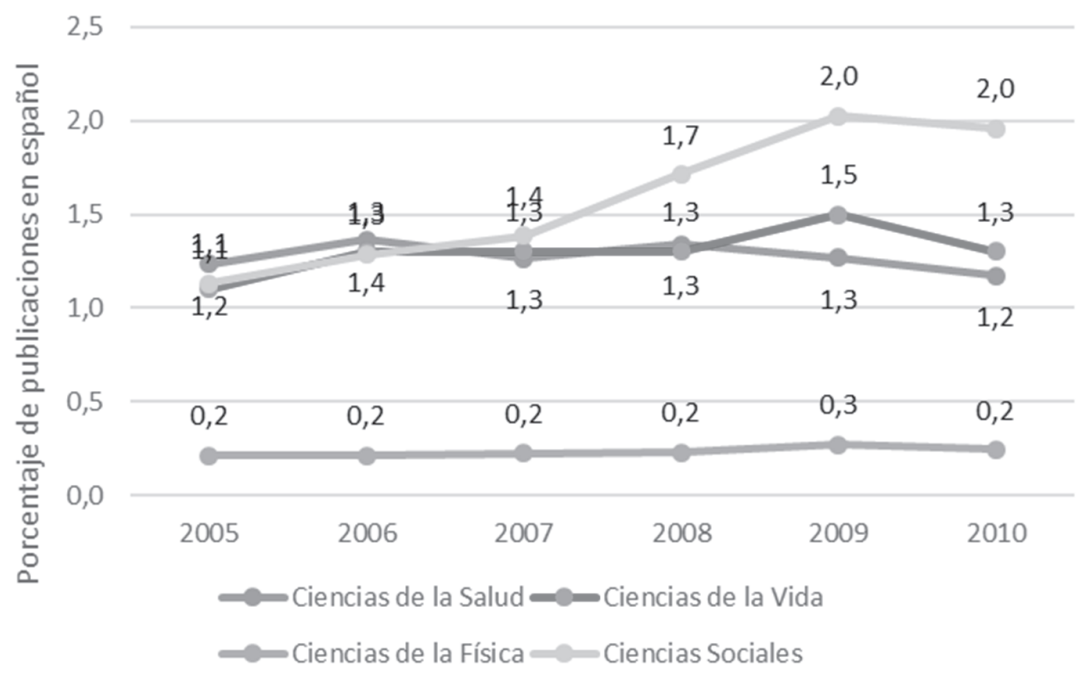

Figura 21. Evolución de las publicaciones en español en las cuatro grandes áreas de Scopus. Periodo 2005-2010

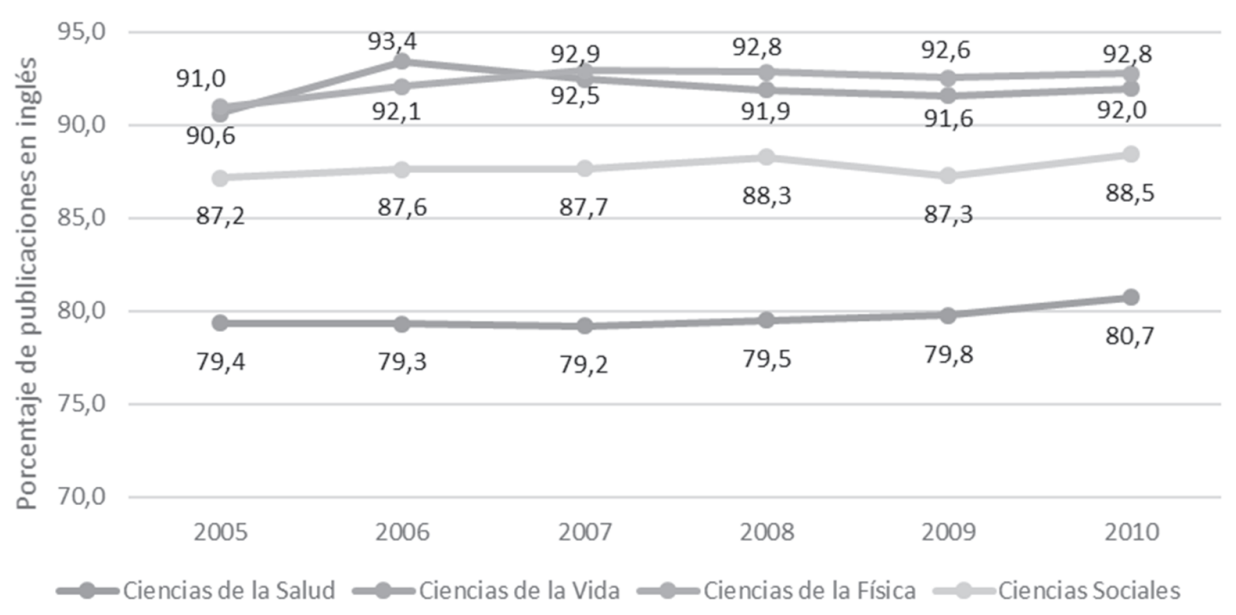

Figura 22. Evolución de las publicaciones en inglés en las cuatro grandes áreas de Scopus. Periodo 2005-2010

La categoría que representa el mayor porcentaje de publicaciones en español es Medicina. Está recogida en dos distintas de Scopus: Ciencias de la Vida y Ciencias de la Salud. En Ciencias de la Vida esta última representa un 50,4 \% 
de las publicaciones en español y un 0,04 \% del total de publicaciones mundiales en esta área. En el área de Ciencias de la Salud, Medicina representa un $81,9 \%$ de las publicaciones en español y un $0,7 \%$ del total de publicaciones en esta categoría. En el área de Ciencias Físicas la de mayor porcentaje de publicaciones en español es Ingeniería con un 20,4 \% de las publicaciones, que representa un 1,4\% del total de publicaciones en esta categoría.

En el ámbito de las Ciencias Sociales, la categoría mejor representada es la denominada Ciencia Social, con un 36,6 \% de las publicaciones en español y un $1,3 \%$ de las publicaciones mundiales.

La producción científica en español: principales indicadores a escala internacional: análisis a través de Inspec

Inspec es una base de datos que recoge publicaciones especializadas en Física, Electrónica, Ingeniería, Informática, Control de producción, Tecnologías de la Información y con una cobertura especial de áreas como Ciencias de los Materiales, Nanotecnología, Oceanografía, Ingeniería Nuclear, Geofísica, Ingeniería Biomédica y Biofísica. Esta base de datos recoge más de 11 millones de registros bibliográficos, incluidos en 5000 revistas científicas y técnicas.

El idioma más utilizado en trabajos científicos recogidos en esta base de datos es el inglés, que representa el 89,7 \% de las publicaciones (tabla 3). Hay que destacar la importancia que en esta base de datos toma el chino frente a lo observado en otras bases de datos multidisciplinares, ya que se convierte en el segundo idioma más utilizado, llegando a representar un $8,2 \%$ de las publicaciones en revistas cubiertas por Inspec durante el periodo 2005-2010 (fig. 23). El español representa un $0,06 \%$ de las publicaciones, dato muy inferior a lo observado en las otras bases de datos multidisciplinares (fig. 24). El área más importante es Informática, que representa el $52 \%$ de las publicaciones en español en Inspec y un 0,09\% de las publicaciones mundiales en esta categoría.

\begin{tabular}{|l|rr|}
\hline \multicolumn{1}{|c|}{ IDIOMA DE PUBLICACIÓN } & \multicolumn{2}{|c|}{ NúMERO DE PUBLICACIONES } \\
\hline Inglés & 3168375 & $(89,70 \%)$ \\
\hline Español & 2069 & $(0,06 \%)$ \\
\hline Publicaciones totales en Inspec & 3529396 & \\
\hline
\end{tabular}

Tabla 3. Porcentaje de publicación en idioma inglés y español en Inspec 


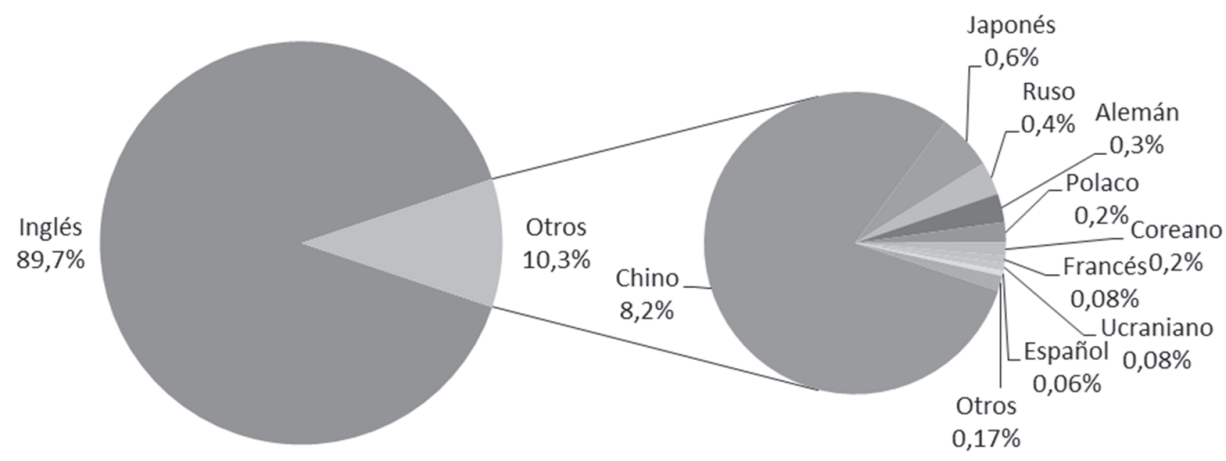

Figura 23. Idiomas de publicación de los trabajos científicos recogidos en Inspec. Periodo 2005-2010

La evolución del uso del inglés como idioma de publicación de artículos científicos en Inspec muestra una tendencia decreciente a lo largo de todo el periodo estudiado, pasando de un $92,5 \%$ en 2005 a un $89 \%$ de los artículos en 2010 (fig. 24). El español muestra una evolución positiva; sin embargo, no llega a superar el $1 \%$ de todas las publicaciones recogidas en esta base de datos.

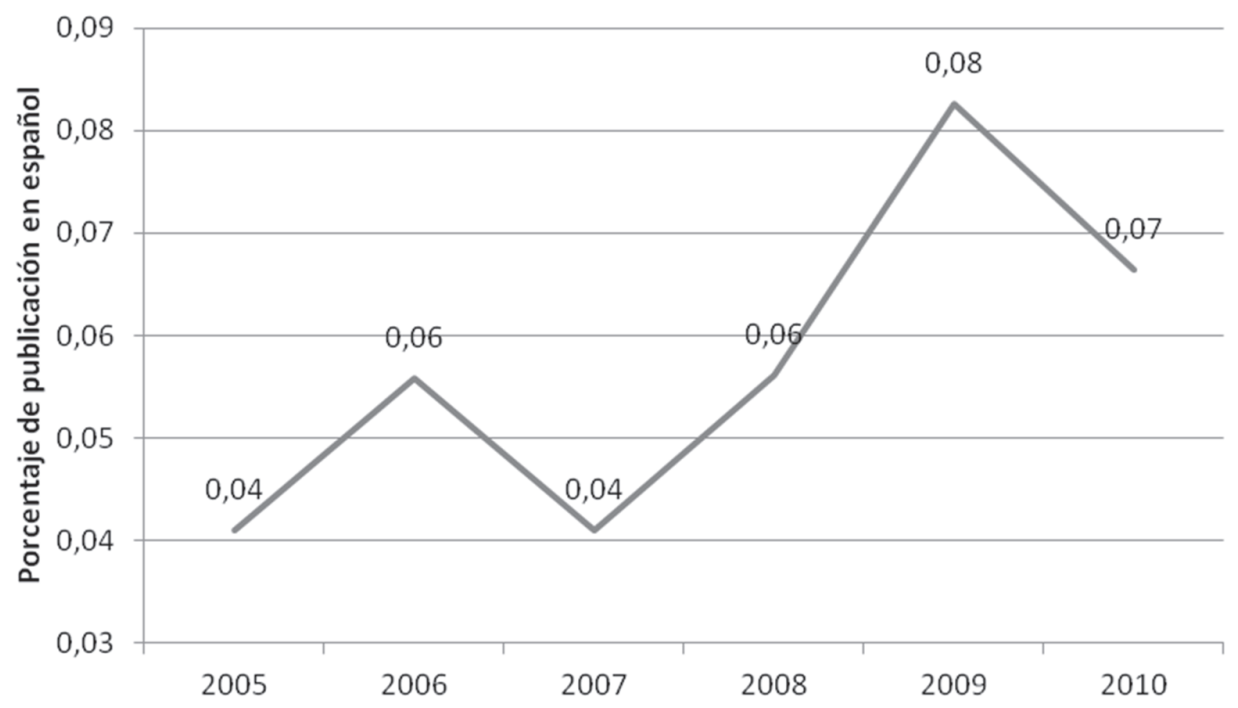

Figura 24. Evolución del porcentaje de publicaciones en español recogidas en Inspec. Periodo 2005-2010 


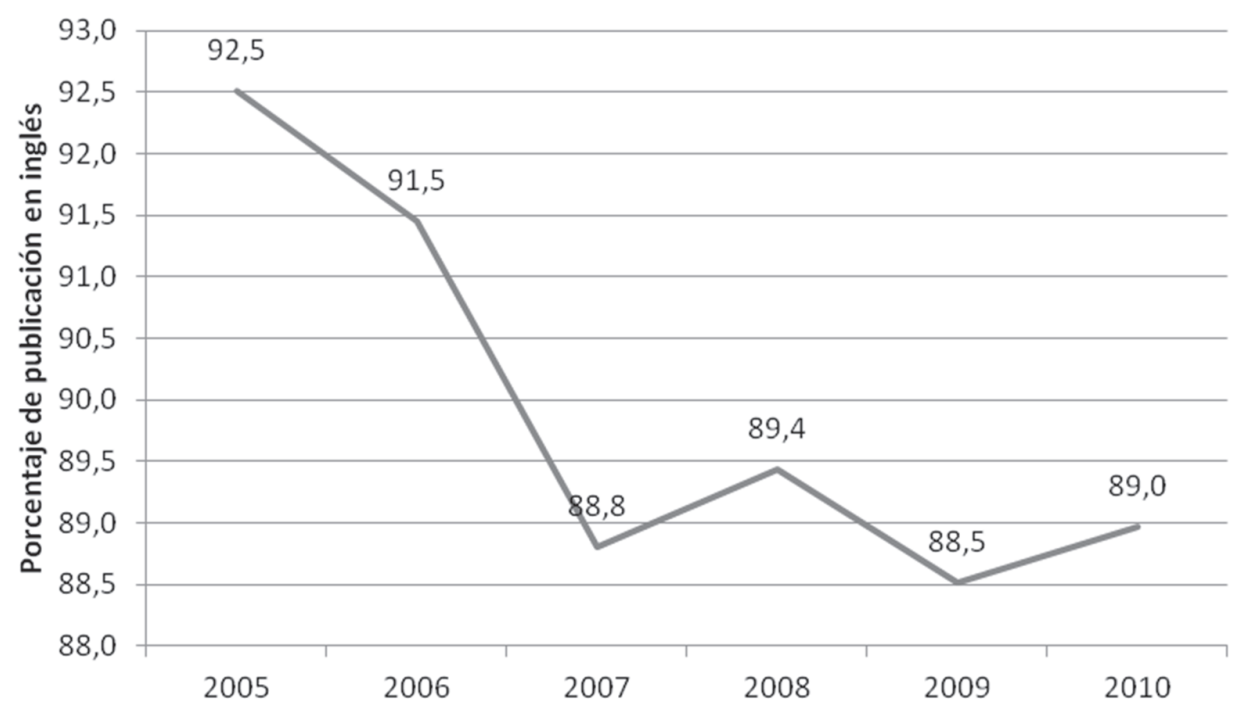

Figura 25. Evolución del porcentaje de publicaciones en inglés recogidas en Inspec. Periodo 2005-2010

La producción científica en español: principales indicadores a escala internacional: análisis a través de PubMed

PubMed es una base de datos de registros bibliográficos especializada en literatura biomédica de áreas como Medicina, Enfermería, Odontología, Veterinaria o Ciencias Preclínicas. Durante el periodo 2005-2010, esta base de datos ha recogido 4821896 publicaciones.

\begin{tabular}{|l|rr|}
\hline \multicolumn{1}{|c|}{ IDIOMA DE PUBLICACIÓN } & \multicolumn{2}{c|}{ NúMERO DE PUBLICACIONES } \\
\hline Inglés & 4442849 & $(92,10 \%)$ \\
\hline Español & 2069 & $(0,75 \%)$ \\
\hline Publicaciones totales en PubMed & 4821896 \\
\hline
\end{tabular}

Tabla 4. Número de publicaciones en PubMed

El idioma de publicación preferente es el inglés, con un 92,1 \% de las publicaciones recogidas en PubMed, con una evolución positiva durante el periodo 2005-2010. El peso del español es muy pequeño, por debajo del $1 \%$ del total de publicaciones, pero muy parecido al de otros idiomas como el alemán o el francés (fig. 26). 
La evolución del número de publicaciones es irregular, con un descenso del porcentaje en 2006 hasta el 0,6\% de publicaciones en español. Desde 2006 hasta 2008, este porcentaje permanece constante; en 2009, aumenta hasta el 1 \% (fig. 27). La disciplina con mayor número de publicaciones en español es la relacionada con el Cáncer, que representa el 27,6 \% de todas las publicaciones en español durante el periodo analizado. Frente a esta evolución del español, el inglés muestra un crecimiento muy regular a lo largo del periodo considerado (fig. 28).

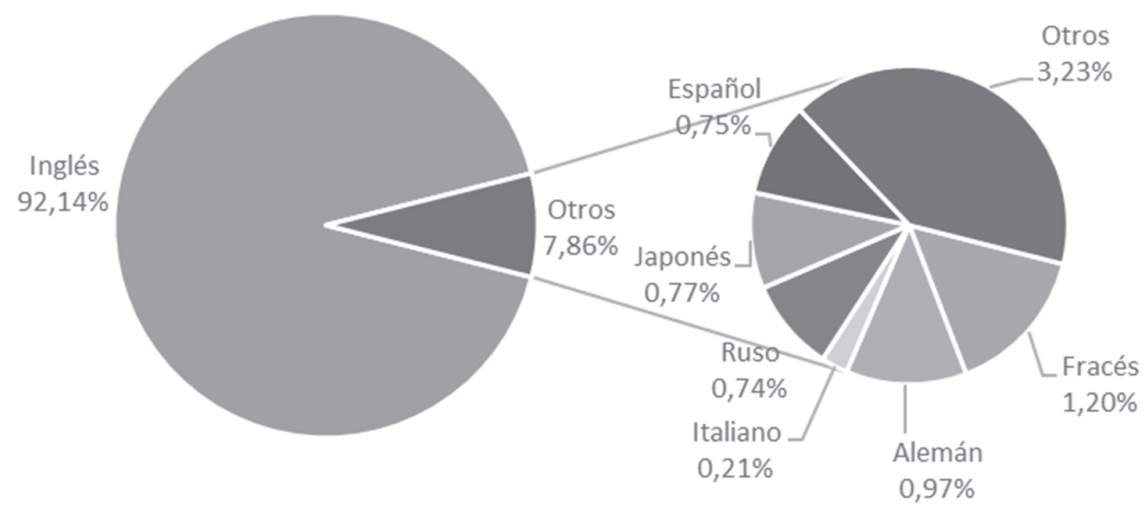

Figura 26. Idiomas de publicación de los trabajos científicos recogidos en PubMed. Periodo 2005-2010

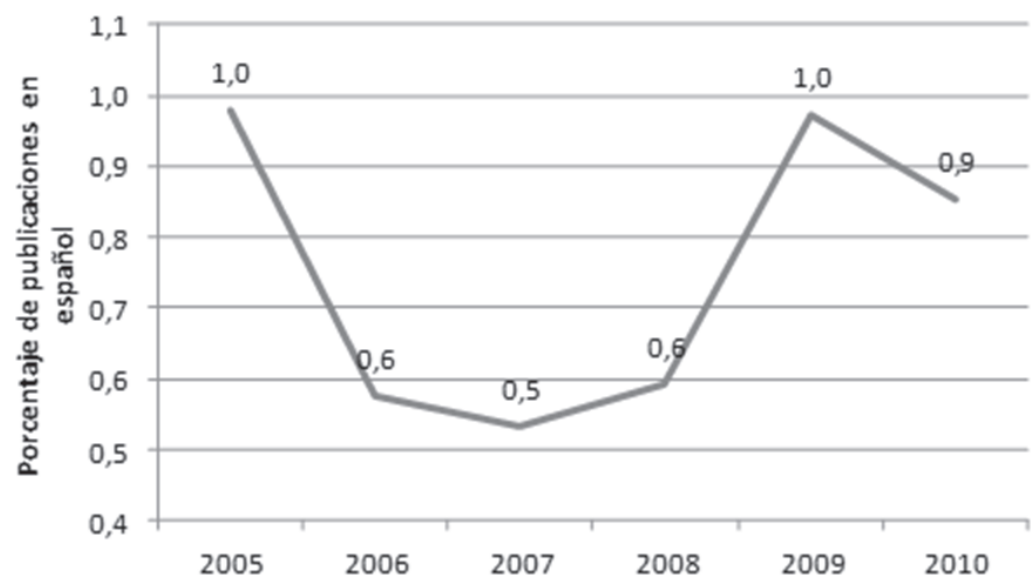

Figura 27. Evolución del porcentaje de publicaciones recogidas en PubMed en español. Periodo 2005-2010 


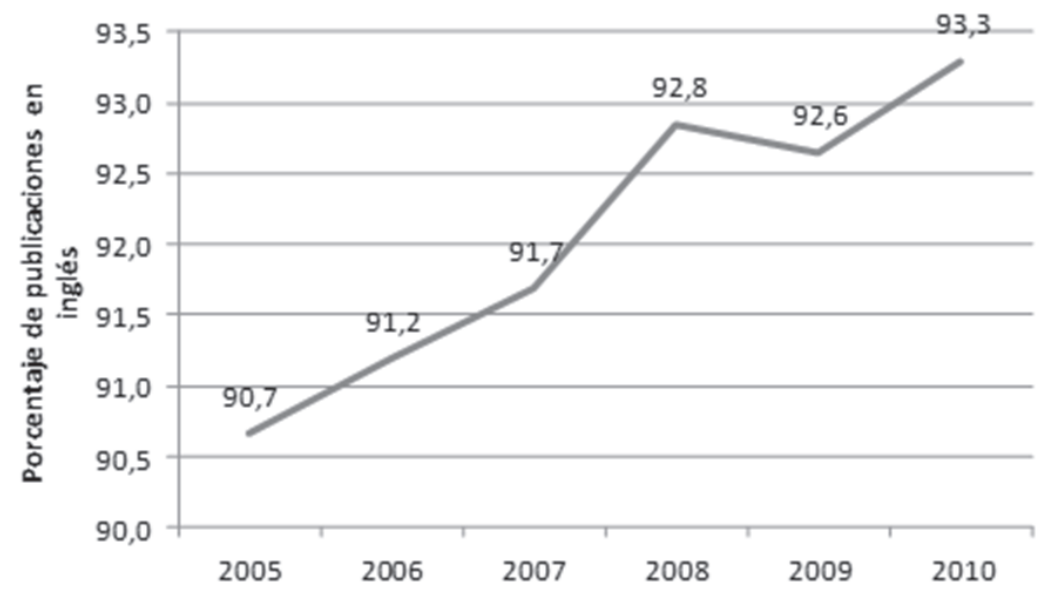

Figura 28. Evolución del porcentaje de publicaciones recogidas en PubMed en inglés. Periodo 2005-2010

\section{CONCLUSIONES}

Los indicadores de producción científica obtenidos en este estudio han permitido cuantificar hasta qué punto el inglés se ha extendido y consolidado como idioma científico a escala mundial. Sin embargo, y considerando el ámbito de las publicaciones como el elemento más representativo de la importancia de las lenguas en la comunidad científica, cabe señalar algunos aspectos sobre el papel del español en la ciencia.

En primer lugar, conviene señalar que, utilizando el sistema público español de I+D como área de análisis, existe un sólido sector editorial científico en el que abunda la edición y distribución de revistas especializadas de notable calidad (Giménez Toledo). Estas revistas, mayoritariamente dirigidas a investigadores españoles, están fuertemente orientadas a servir de vehículos de comunicación en disciplinas científicas ligadas a intereses territoriales o locales, como es el caso de la investigación sobre aspectos biológicos o geológicos de nuestro territorio (Rey/Martín/Plaza), así como a otras disciplinas vinculadas a actividades e intereses socioeconómicos, como es el caso de las publicaciones de carácter tecnológico. Prácticamente todas las revistas que responden a este tipo de planteamientos son editadas en lengua española, al menos en gran parte (Plaza y otros 2009).

Al margen de la dimensión local antes aludida y ampliando la perspectiva de análisis a una escala global, conviene destacar que el español es, en cier- 
ta medida, un idioma especialmente importante en algunos ámbitos disciplinares incluidos entre las Ciencias Sociales, y más especialmente en algunas disciplinas y subdisciplinas propias de las Humanidades. Entre estas últimas, la Literatura es una disciplina en la que se contabiliza un buen número de contribuciones en español, entendiendo que es precisamente la lengua española y todo lo que desde un punto de vista científico atañe a la producción literaria en español lo que hace especialmente relevante a nuestro idioma. Por otra parte, los estudios lingüísticos sobre el español también tienen una amplia presencia entre las revistas editadas en lengua española.

Entre los estudios de carácter histórico, social y económico, que conciernen al ámbito geopolítico y cultural latinoamericano, el español, como cabe suponer, tiene un valor muy representativo como lengua vehicular, si bien este entorno es multidisciplinar y su identificación y valoración cuantitativa implicaría un análisis específico que desborda los límites establecidos para este estudio.

En cualquier caso, la importancia de los estudios europeos sobre América Latina se ve reflejada en la existencia de diversas redes de investigación, así como en redes de información científica que, como es el caso de REDIAL (Red Europea de Información y Documentación sobre América Latina), tiene como objetivo dar seguimiento a la investigación sobre los sistemas de Información en Ciencias Sociales y Humanas sobre América Latina en Europa. A nivel latinoamericano también existen iniciativas de gran importancia para la difusión de la producción científica regional, publicada principalmente en idioma español. Un ejemplo es Latindex, cuyos datos se analizaron en este artículo. Otro ejemplo es Scielo, una biblioteca electrónica que surgió como iniciativa de FAPESP y BIREME en Brasil y que permite la publicación electrónica de ediciones completas de revistas científicas. Hoy en día cuenta con más de mil revistas a texto completo y de acceso abierto, procedentes de quince países iberoamericanos.

El español también tiene una fuerte presencia en algunas disciplinas de carácter experimental, fundamentalmente en el ámbito de la investigación en Ciencias de la Salud. En este contexto, la medicina clínica es, para los intereses científicos de los países iberoamericanos, un ámbito en el que, además de contar con revistas editadas en español, la información va dirigida a unos colectivos especializados en los que esta es la lengua de trabajo habitual. En este contexto, hay una serie de subdisciplinas en las que, a escala internacional, el español es muy relevante, como es el caso de la Medicina General e Interna, 
la Ética Médica, la Urología y Nefrología, la investigación sobre el sistema respiratorio, la Nutrición, la Dietética y la Pediatría.

Por otra parte, y en este mismo contexto de las Ciencias Experimentales, el español tiene una significativa presencia en disciplinas tales como Paleontología y Micología. A caballo entre las Ciencias Experimentales y las Ciencias Sociales, la Arqueología, por cuanto corresponde a la investigación en Iberoamérica, es una disciplina con un significativo número de artículos en español.

En el ámbito de las Tecnologías, y a escala internacional, destaca la producción científica en tecnologías de la Construcción y Robótica.

Superando el concepto de producción científica (entendido como conjunto de trabajos publicados en revistas científicas), el peso del español en ciencia es mucho más importante de lo que en una primera lectura se deriva de los indicadores obtenidos en este estudio (Plaza y otros 1999 y 2013). Existen ámbitos de actividad donde el uso del español es determinante, como son las actividades docentes, la divulgación de la ciencia y el intercambio de ideas y conocimientos entre investigadores de países de habla española. De hecho, la formación de los investigadores en estos países, tanto en su etapa universitaria como en etapas posteriores a la realización de estudios de doctorado, se realiza en gran medida usando el español como lengua de trabajo. La labor diaria en el laboratorio o en el centro de investigación, así como la participación en congresos, seminarios y en general en reuniones científicas nacionales celebradas en países de lengua española se realizan mayoritariamente en este idioma.

Junto a la comunicación en ciencia, independientemente de la comunidad profesional a la que va dirigida la información, no conviene olvidar el importantísimo papel que también juega nuestra lengua en la transmisión social de conocimientos y aplicaciones de las tecnologías. Fundamentalmente de aquellas cuyo desarrollo está fuertemente ligado a los avances de la investigación científica.

A todo lo anterior, hay que añadir el enorme interés que, para la comunidad de habla española, tiene la divulgación de la ciencia y la tecnología a través de revistas divulgativas y otros medios de comunicación. La transmisión de conocimientos científicos altamente especializados y su conversión en un lenguaje comprensible para el lector medio y para cualquier tipo de público no familiarizado con los lenguajes de la ciencia juega un papel fundamental en la captación del interés por la investigación científica y por un mejor conocimiento y aceptación social de las aplicaciones de la ciencia en prácticamente todos los ámbitos de actividad. 
El correcto uso del español en directrices, normas, legislación, informes técnicos, económicos y políticos, patentes y otros documentos de interés en ciencia y tecnología es esencial para una adecuada información por parte de aquellos agentes que contribuyen activamente en las distintas actividades inherentes a los sistemas de investigación y desarrollo, tanto en su vertiente pública como privada.

Teniendo en cuenta lo anteriormente expuesto en este apartado, resulta obligado destacar el importante papel de los especialistas que trabajan en el ámbito de la terminología científica en español, ya que este idioma presenta serias carencias en el ámbito de la terminología científica, carencias que en parte se deben a la utilización generalizada del inglés en ciencia y tecnología y a la constante irrupción de nuevos términos en inglés para los que, en su mayoría, no existe una correcta traducción al español. El uso de términos y expresiones científicas en inglés viene siendo habitual en las diferentes formas de transmisión del conocimiento utilizadas por investigadores españoles e iberoamericanos. Siendo el español una lengua hablada por unos 450 millones de personas, es del máximo interés disponer de una terminología científica en español, actualizada y con sus correspondientes equivalencias en lengua inglesa (Vera Torres).

Por último, es de capital importancia reconocer el muy importante papel que juegan los especialistas en información y documentación científica en actividades tales como la puesta a punto y distribución de productos y servicios documentales donde el uso del español es un factor común y de interés específico para la comunidad investigadora de habla española y para otros demandantes de información científica y tecnológica en español. Entre estos productos y servicios, que en gran medida tienen una significativa variable de mercado, destacan las bibliotecas, las bases de datos y los repositorios de bibliografía y documentación científica, las sedes web y sus correspondientes contenidos en lengua española, las herramientas informáticas, los tesauros, vocabularios, índices y otros lenguajes documentales.

Como se ha señalado antes, pese al limitado peso del español en la arena científica internacional, son muchas las actividades científicas que se vehiculan en lengua española. Su interés económico trasciende al valor que pueda estimarse para las operaciones económicas ligadas a las tareas de investigación científica en España y países hispanoamericanos: adquisición y mantenimiento de infraestructuras, formación de nuevos investigadores, costes de personal científico, financiación de proyectos, redes y centros, etc. La investigación científica y el desarrollo de nuevas tecnologías son, si no los únicos, los prin- 
cipales activos con que se cuenta para el futuro. Las sociedades basadas en el conocimiento científico deben aprovecharse de este en todas sus posibilidades y formas de expresión. La lengua en ciencia, y en particular el español, pese a su dimensión transversal, no puede dejar de ser percibida como una variable social y económica de primer orden.

El objetivo final que ha servido de guía para este estudio ha sido el de contribuir, aunque sea a una escala limitada, al mejor conocimiento y comprensión del valor real y potencial del español en el mundo científico, para la adecuada utilización de este recurso para el desarrollo de nuestras sociedades, la correcta explotación de los recursos naturales y culturales y la conveniente protección de nuestro entorno.

\section{OBRAS CITADAS}

Abejón Peña, Teresa, Ángeles Maldonado Martínez, Luis Rodríguez Yunta y M. ${ }^{a}$ Cruz Rubio Liniers. "La base de datos ISOC como sistema de información y fuente para el análisis de las ciencias humanas y sociales en España”. El Profesional de la Información 18.5 (2009): 521-28.

Alonso Gamboa, José Octavio y Jane M. Russell. "Latin American Scholarly Journal Databases: A Look Back to the Way Forward". Aslib Proceedings 64.1 (2012): 32-45.

BOE: Boletín Oficial del Estado. "Ley 14/2011 de 1 de junio, de la Ciencia, la Tecnología y la Innovación”. BOE 131 de 2 de junio de 2011. 54387-455.

FECYT: Reyes Sequera, ed. Ciencia, tecnología y lengua española: la terminología científica en español. Madrid: Fundación Española para la Ciencia y la Tecnología, 2004.

Giménez Toledo, Elea. "Reflexiones a partir de la jornada de FECYT sobre las medidas de apoyo a las revistas científicas españolas". Revista Española de Documentación Científica 30.2 (2007): 257-60.

Plaza, Luis M. "El idioma español en la comunidad científica internacional". Contrastes 39 (2005): 112-15.

Plaza, Luis M. y María Bordons. "Proyección internacional de la ciencia española". Enciclopedia del español en el mundo: anuario del Instituto Cervantes 2006-2007. Madrid: Instituto Cervantes, 2006. 547-67.

Plaza, Luis M., E. Fernández, A. Román y C. Ruiz. "Presencia del español en la producción científica". Anuario del Instituto Cervantes 1999. Madrid: Instituto Cervantes, 1999. 23-64. 
Plaza, Luis M., Begoña Granadino y María José Arias-Salgado. "Las revistas científicas editadas en lengua española: su misión actual y sus perspectivas como instrumento para la difusión internacional de la ciencia”. El español, lengua para la ciencia y la tecnología: presente y perspectivas de futuro. Coord. Verónica Vivanco Cerbero. Madrid: Instituto Cervantes, 2009. 41-56.

Plaza, Luis M., Begoña Granadino y Esther García-Carpintero. "Estudio bibliométrico sobre el papel del español en ciencia y tecnología". El español como lengua de comunicación científica. Madrid: Fundación telefónica/Editorial Ariel, 2013. 315-66.

REDIAL y CEISAL. América Latina Portal Europeo. 1 de agosto de 2017. <http:// www.red-redial.net/>.

Rey, Jesús, María José Martín y Luis M. Plaza. “The Role of Domestic Journals in Disseminating Research Results in Scientific Disciplines of Marked Local, Regional or National Interest: The Case of Spanish Journals on Earth Sciences". Proceedings of the 7th Conference of the ISSI. Ed. César A. Macías-Chapula. Colima: Universidad de Colima, 1999. 590-91.

Vera Torres, Juan Antonio. "La terminología científica en español: análisis de la situación y proyectos de actuación futura". III Acta Internacional de la Lengua Española. 2008. 31 de julio de 2017. <http://www.rac.es/ficheros/ doc/00498.pdfs. 